\title{
Alterations of hemostatic parameters in the early development of allogeneic hematopoietic stem cell transplantation-related complications
}

\author{
Yue Han • Li Zhu • Aining Sun • Xiaoxu Lu • Luping Hu • Lili Zhou • Yongya Ren • \\ Xiaohui Hu $\cdot$ Xiaojin Wu - Zhaoyue Wang $\cdot$ Changgeng Ruan $\cdot$ Depei Wu
}

Received: 11 September 2010 / Accepted: 5 June 2011 /Published online: 15 June 2011

(C) The Author(s) 2011. This article is published with open access at Springerlink.com

\begin{abstract}
Thrombotic events are common and potentially fatal complications in patients receiving hematopoietic stem cell transplantation (HSCT). Early diagnosis is crucial but remains controversial. In this study, we investigated the early alterations of hemostatic parameters in allogeneic HSCT recipients and determined their potential diagnostic values in transplantation-related thrombotic complications and other post-HSCT events. Results from 107 patients with allogeneic HSCT showed higher levels of plasma plasminogen activator inhibitor-1 (PAI-1), fibrinogen, and tissue-plasminogen activator (t-PA) and a lower level of plasma protein $\mathrm{C}$ after transplantation. No change was found for prothrombin time, antithrombin III, D-dimer, and activated partial thromboplastin time following HSCT. Transplantation-related complications (TRCs) in HSCT patients were defined as thrombotic $(n=8)$, acute graft-versus-host disease (aGVHD, $n=45)$, and infectious $(n=38)$. All patients with TRCs, especially the
\end{abstract}

Y. Han $(\bowtie) \cdot$ A. Sun $\cdot$ X. Lu $\cdot$ L. Hu $\cdot$ L. Zhou $\cdot$ Y. Ren $\cdot$ X. Hu $\mathrm{X}$. Wu $\cdot \mathrm{Z}$. Wang $\cdot$ C. Ruan $\cdot \mathrm{D}$. Wu $(\square)$

Department of Hematology, Jiangsu Institute of Hematology,

The First Affiliated Hospital of Soochow University,

No.188. Shi Zi Street,

Suzhou 215006, People's Republic of China

e-mail: sdfyyxyk@yahoo.com

e-mail: wudepei@medmail.com.cn

Y. Han $\cdot$ A. Sun $\cdot$ X. Lu $\cdot$ L. Hu $\cdot$ L. Zhou $\cdot$ Y. Ren $\cdot$ X. Hu $\cdot$

$\mathrm{X}$. Wu $\cdot$ Z. Wang $\cdot$ C. Ruan $\cdot$ D. Wu

Key Laboratory of Thrombosis and Haemostasis,

Ministry of Health,

Suzhou 215007, People's Republic of China

L. Zhu

Cyrus Tang Hematology Center, Jiangsu Institute of Hematology,

The First Affiliated Hospital of Soochow University,

Suzhou 215123, People's Republic of China patients with thrombotic complications, presented significant increases in the mean and maximum levels of PAI-1 during the observation period. Similarly, a high maximum t-PA level was found in the thrombotic group. In contrast, apparent lower levels of mean and minimum protein $\mathrm{C}$ were observed in the TRC patients, especially in the aGVHD group. Therefore, the hemostatic imbalance in the early phase of HSCT, reflecting prothrombotic state and endothelial injury due to the conditioning therapy or TRCs, might be useful in the differential diagnosis of the thrombotic complication from other TRCs.

Keywords Thrombotic complication · Transplantationrelated complications (TRCs) - Plasminogen activator inhibitor-1 (PAI-1) · Protein C (PC) · Hematopoietic stem cell transplantation (HSCT)

\section{Introduction}

Allogeneic hematopoietic stem cell transplantation (HSCT) is an effective strategy for a variety of hematological disorders [1-3]. Improvements in human leukocyte antigen typing and increased experience in managing transplantation-related complications (TRCs) have resulted in decreased morbidity and mortality following HSCT during the last decade [4-6]. Nonetheless, thrombotic events are recognized as the common and potentially fatal complications in HSCT recipients, such as hepatic veno-occlusive disease (VOD), transplantation-related thrombotic microangiopathy (TATMA), catheter-associated thrombosis, and deep vein thrombosis (DVT). It is generally assumed that endothelium damage and coagulation disturbance induced by pretransplant conditioning regimens or some other factors may contribute to the development of thrombotic events [7-9]. 
However, their early diagnosis remains controversial since their clinical manifestations are non-specific and may be similar to a number of other TRCs, such as acute graftversus-host disease (aGVHD) and infection. Therefore, prompt finding of thrombotic events is essential for the differential diagnosis and effective early therapy.

Many hemostatic abnormalities have been reported in various thrombotic events following allogeneic HSCT, including fibrinolytic and coagulation parameters [10]. In particular, elevated levels of plasma plasminogen activator inhibitor (PAI-1) antigen have been observed in patients with VOD or TA-TMA [11-15] and serve as one crucial noninvasive tool for the diagnosis of VOD [11]. The changes of other hemostatic parameters, including plasma protein $\mathrm{C}$ (PC), tissue-plasminogen activator (t-PA), antithrombin III (ATIII), and D-dimer (D-Di), have also been described in TRCs $[12,14,16,17]$. However, the early discrimination of thrombotic event from other complications has not been successfully defined. We conducted a prospective survey to investigate the correlation of hemostatic parameters with TRCs at predetermined time points to evaluate their values in the differential diagnosis of TRCs.

\section{Patients and methods}

Patients and transplant procedure

One hundred and seven patients (67 males and 40 females) receiving allogeneic HSCT from May 2006 to August 2009 were enrolled in this study, with a median age of 33 years (range, 10-59), including eight patients under 18 years old (8/ $107,7.5 \%$ ). Patients were admitted for a variety of hematologic disorders at various disease stages. Human leukocyte antigen (HLA) typing of the recipients and donors was performed by high-resolution DNA typing using polymerase chain reaction sequence-specific oligonucleotides for both HLA class I (HLA-A, HLA-B, HLA-C) and class II (HLADRB1, HLA-DQB1) loci. Matching was defined as 10/10 fully matched. The patients and transplant characteristics were summarized in Table 1. All patients or their legal representatives signed their informed consents, and the local ethics committee approved this study.

All patients were nursed in laminar airflow rooms and received a standard regimen for the prevention of infectious complications (non-absorbable antibiotics for gut decontamination, ganciclovir and cotrimazole for virus and Pneumocystis infections, fluconazole for fungal prophylaxis, and hyperhydration and mesna for the prevention of cyclophosphamide-induced hemorrhagic cystitis). All cellular blood products were leukocyte-depleted and irradiated prior to transfusion. All allogeneic patients received prophylactic therapy with cyclosporine and methotrexate for
Table 1 The characteristics of patients

\begin{tabular}{lc}
\hline Characteristics & Patients (\%) \\
\hline Total & 107 \\
Sex & \\
Male & $67(62.6)$ \\
Female & $40(37.7)$ \\
Median age (years, range) & $33(10-59)$ \\
Underlying disease & \\
Chronic myeloid leukemia & $33(30.7)$ \\
Acute myeloid leukemia & $39(36.4)$ \\
Acute lymphoblastic leukemia & $21(19.6)$ \\
Lymphoma & $8(7.5)$ \\
Others & $6(5.3)$ \\
Type of transplant & \\
Matched unrelated donor & $24(22.8)$ \\
Matched related donor & $75(70.1)$ \\
Mismatched related donor & $8(7.0)$ \\
Conditioning regimen & \\
Bu-Cy & $75(70.1)$ \\
TBI-Cy & $23(21.5)$ \\
Flu-Cy-ATG & $6(5.3)$ \\
Others & $3(2.6)$ \\
Stem cells infused CD34 ${ }^{+}\left(10^{6} / \mathrm{kg}\right.$, range) & $3.82(0.03-21.63)$ \\
ANC engraftment (days, range) & $12(10-20)$ \\
\hline
\end{tabular}

$B u$ busulfan, $C y$ cyclophosphamide, TBI total body irradiation, Flu fludarabine, ANC engraftment day on which the absolute neutrophil count was $>0.5 \times 10^{9} / 1$

GVHD, with a continuous i.v. infusion of cyclosporine $(3 \mathrm{mg} / \mathrm{kg}$ ) over $24 \mathrm{~h}$ starting on day -2 and i.v. administrations of methotrexate with the dose of $15 \mathrm{mg} / \mathrm{m}^{2}$ on day 1 and $10 \mathrm{mg} / \mathrm{m}^{2}$ on days 3,6 , and 11 (total dose, $45 \mathrm{mg} / \mathrm{m}^{2}$ ). Unrelated and mismatched patients also received antithymocyte globulin (ATG) and mycophenolate mofetil (MMF) for GVHD prophylaxis, with ATG (Rabbit Anti-human Thymocyte Globulin, IMTIX-SANGSTAT, France) $(2.5 \mathrm{mg} / \mathrm{kg}$ once daily i.v.) administered from days -5 to -3 and MMF $(15 \mathrm{mg} / \mathrm{kg}$ twice daily p.o.) starting on day -10 . For prevention of thrombotic complications, a continuous i.v. infusion of low-dose heparin $(100 \mathrm{U} / \mathrm{kg})$ over $24 \mathrm{~h}$ and a three-time daily i.v. prostaglandin E1 $(10 \mu \mathrm{g})$ were administered from days -10 to 28 . Total parenteral nutrition was given if indicated.

\section{Classification of TRCs}

Patients were evaluated once a week for TRC, and the occurrence of aGVHD, VOD, TMA, DVT, and infection was described in Table 2. aGVHD was assessed and graded by clinical and biological criteria according to Klingebiel and Schlegel [18]. 
Table 2 Transplantation-related complications

\begin{tabular}{lccc}
\hline Complications & \multicolumn{2}{c}{ Patients (\%) } & \\
\cline { 2 - 4 } & Total TRCs & First-onset TRCs & Single TRCs \\
\hline Acute GVHD & $45(42.1)$ & $38(35.5)$ & $22(20.6)$ \\
Grade 1 & $23(21.5)$ & $21(19.6)$ & $14(13.1)$ \\
$\geq$ Grade 2 & $22(20.6)$ & $17(15.9)$ & $8(7.5)$ \\
Thrombotic complications & $8(7.5)$ & $7(6.5)$ & $3(2.8)$ \\
VOD & $6(5.6)$ & $5(4.7)$ & $2(1.9)$ \\
TMA & $1(0.9)$ & $1(0.9)$ & $1(0)$ \\
DVT & $1(0.9)$ & $1(0.9)$ & $20(18.7)$ \\
Infection & $38(35.5)$ & $28(26.2)$ & \\
$\geq 2$ complications & $28(26.2)$ & & \\
No complication & $34(31.8)$ & & \\
\hline
\end{tabular}

VOD was diagnosed according to Seattle clinical criteria [19], as having two of the following before day 30: (1) hyperbilirubinemia (bilirubin $\geq 34.2 \mu \mathrm{mol} / \mathrm{l}$ ), (2) painful hepatomegaly, and (3) unexplained weight gain ( $>2 \%$ from the baseline), and no other explanation could be present for these signs and symptoms. The diagnosis was also confirmed by Doppler ultrasound or liver biopsy in all cases.

TMA was defined as the fulfilment of the following definition proposed by the Blood and Marrow Transplant Clinical Trials Network [20]: (1) RBC fragmentation with two schistocytes per high-power field on peripheral smear, (2) concurrent increased serum LDH above institutional baseline, (3) concurrent renal and/or neurologic dysfunction without other explanations, and (4) negative direct and indirect coombs' test results. Diagnosis of DVT was based upon a positive result of ultrasound or venography.

Infectious complications were defined by clinical and laboratory parameters [21]: (1) body temperature $\geq 38.5^{\circ} \mathrm{C}$ on a single occasion or $\geq 38^{\circ} \mathrm{C}$ on two separate occasions among 6-h intervals; (2) exclusion of other fever origins, effective antibiotic therapy; (3) positive microorganisms in blood, sputum, or urine cultures.

Assays of hemostatic parameters

Venous blood was drawn weekly in the morning from 2 weeks (week -2) prior to stem cell infusion (week 0) until 4 weeks after transplantation (week +4 ) and was anticoagulated with 1:9 volumes of $3.8 \%$ sodium citrate. Plasma samples were obtained by centrifugation within $1 \mathrm{~h}(3,000 \times \mathrm{g}$ for $15 \mathrm{~min})$ for complete platelet removal. Aliquots were frozen and stored at $-80^{\circ} \mathrm{C}$ until assayed.

Plasma levels of various hemostatic parameters were monitored by standard techniques. PAI-1, t-PA, and PC antigens were determined by enzyme-linked immunosorbent assay (Shanghai Sun Biotech, Shanghai, China) following the manufacturer's instructions (normal ranges, 5-45 ng/ml for
PAI-1, 1.0-12 ng/ml for t-PA, 3.0-5.2 $\mu \mathrm{g} / \mathrm{ml}$ for PC). Activated partial thromboplastin time (APTT; normal range, 28-40 s), prothrombin time (PT; normal range, 11-14.5 s), fibrinogen ( $\mathrm{Fg}$; normal range, 2-5 $\mu \mathrm{g} / \mathrm{ml}$ ), ATIII (normal range, $80-120 \%$ ), and $\mathrm{D}-\mathrm{Di}$ (normal range, $0.01-0.5 \mu \mathrm{g} / \mathrm{ml}$ ) were performed on the hemagglutinin equipment (Diagnostica Stago, Asnieres, France).

Statistical analysis

Statistical analysis was performed using SPSS 11.5 software. The results were expressed as mean \pm standard deviation. Categorical variables were analyzed using the chi-square test and by Mann-Whitney $U$ for group differences. Continuous variables were compared with Student's $t$ test. All tests used an $\alpha$ level of 0.05 , and $P<0.05$ was considered statistically significant.

\section{Results}

Characteristics and complications of patients receiving HSCT

The characteristics of patients were shown in Table 1. Of 107 patients, 24 transplants were from matched unrelated donors, 75 from matched related donors, and 8 from mismatched related donors. The disease status at transplant was not related to the alteration of hemostatic parameters. The pre-transplant conditioning regimen included Bu-Cy in $75(70.1 \%)$ cases, TBI-Cy in 23 $(21.5 \%)$, Flu-Cy-ATG in $6(5.3 \%)$, and others in the remaining $3(2.6 \%)$ cases. Median number of $\mathrm{CD}^{+} 4^{+}$cell infusion was $3.82 \times 10^{6} / \mathrm{kg}$ (range, 0.03-21.63). Median absolute neutrophil count (ANC) engraftment $\left(>0.5 \times 10^{9} / 1\right)$ was 12 days (range, 10-20) with no significant difference among different groups. 
Three groups of TRCs were defined by their corresponding criteria as shown in Table 2. Ninety-one complications occurred in all patients observed, of which 45 met the aGVHD criteria (22 with grades 2-4), 8 were classified as thrombotic complications (6 VOD, 1 TA-TMA, 1 DVT), and 38 were diagnosed as infectious complications. Twenty-eight patients were found to have at least two types of complications, and 34 patients had no complications during the observation period. Based on the first-onset complications following HSCT, 38 patients presented with aGVHD, 28 with infection, and 7 with thrombotic complication. When grouped with single complication, 22 patients were complicated with aGVHD, 3 with thrombosis, and 28 with infection.

\section{Changes of hemostatic parameters during HSCT}

We measured plasma levels of hemostatic parameters in all the patients who underwent HSCT. Results were given as mean \pm 1 standard deviation (SD). Week 0 is defined as the week immediately after stem cell transfusion. As shown in Table 3, although plasma levels of APTT, ATIII, PT, and D-Di were normal, plasma PAI-1, PC, t-PA, and Fg antigen levels were changed in patients receiving HSCT. During conditioning treatment (week -1), a significant increase in PAI-1 and an apparent decrease in PC were observed followed by remarkable diminutions of PAI-1 and t-PA levels and an elevation of PC level immediately after stem cell transfusion (week 0). After grafting, levels of PAI-1 and t-PA increased, while PC decreased in the first week of transplantation and then increased throughout the observation period. In addition, fibrinogen showed a significant increase after transplantation.

\section{Alteration of plasma levels of PAI-1 and PC in patients} with TRCs during HSCT

We next analyzed the correlations of plasma levels of PAI-1 and PC with TRCs in the course of HSCT. Before conditioning therapy, patients with or without complications did not show any significant difference in the mean plasma PAI-1 antigen levels. Their PAI-1 levels increased during the conditioning period $(P<0.05)$, decreased immediately after stem cell transfusion, and then increased after engraftment $(P<0.05)$. However, patients with complications presented with a tendency of increased PAI-1 compared with those without complication during the conditioning period and the first 2 weeks after transplantation $(P<0.05)$. Among them, patients with thrombotic complications showed a more significant increase in plasma PAI-1 throughout the observation period (Fig. 1a). Similar to plasma PAI-1, protein $\mathrm{C}$ antigen levels in patients with or without complications were normal before conditioning (week -2), but dropped during conditioning phase

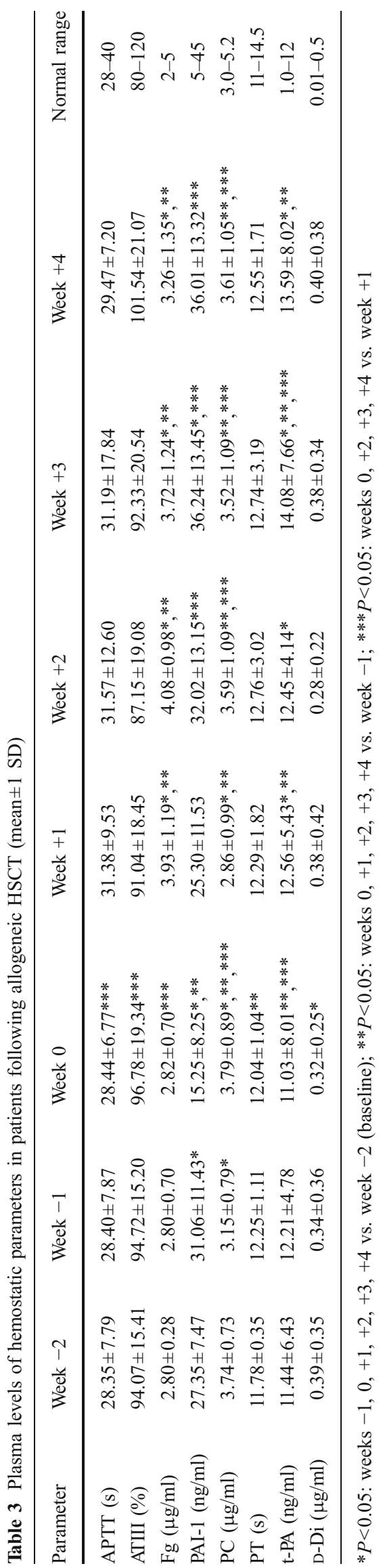


Fig. 1 Plasma levels of PAI-1 and $\mathrm{PC}$ antigens in transplantation-related complications during allogeneic HSCT. Week 0 is defined as the week immediately after stem cell transfusion; a showed that PAI-1 levels (mean $\pm \mathrm{SD}$ ) were elevated significantly in patients following pre-conditioning and transplantation. All the patients with three different complications presented with a tendency of increased PAI-1 compared with those who had no complication $\left({ }^{*} P<0.05\right)$ and showed a remarkable elevation in the patients with thrombotic complications; b showed that visible decreases of PC levels (mean \pm $\mathrm{SD})$ were found following preconditioning and transplantation. All the patients with three different complications presented with lower level of PC compared with those who had no complication $(* P<0.05)$ and showed a remarkable diminution in the patients with aGVHD. $\triangle P<0.05$ : compared with the initial value before conditioning, week -2 . $\# P<0.05$ : compared with the value after stem cell transfusion, week 0

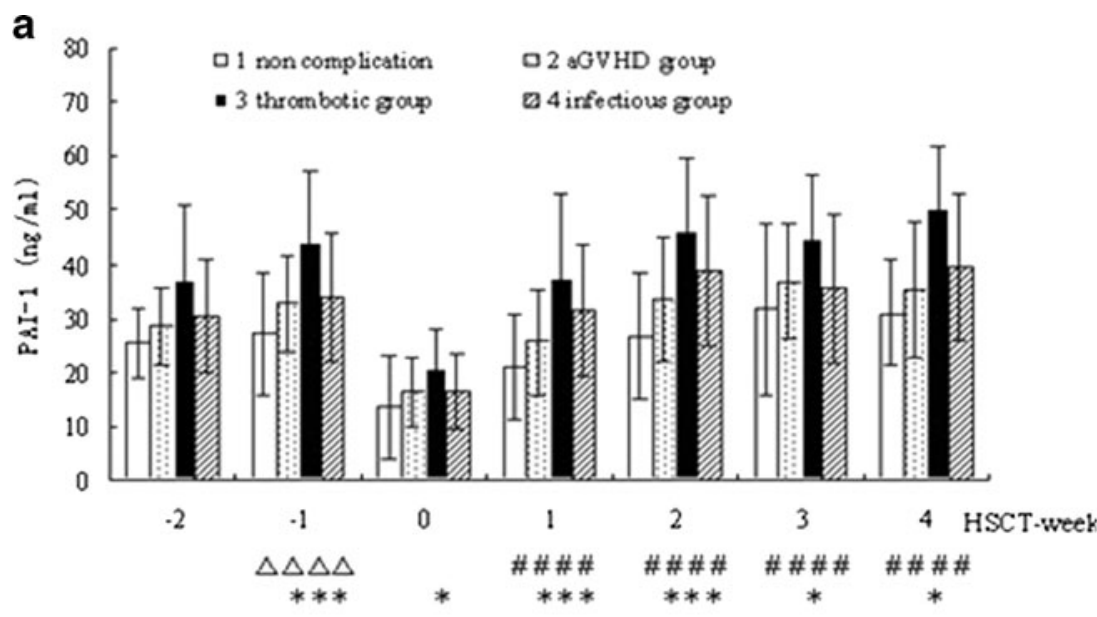

b

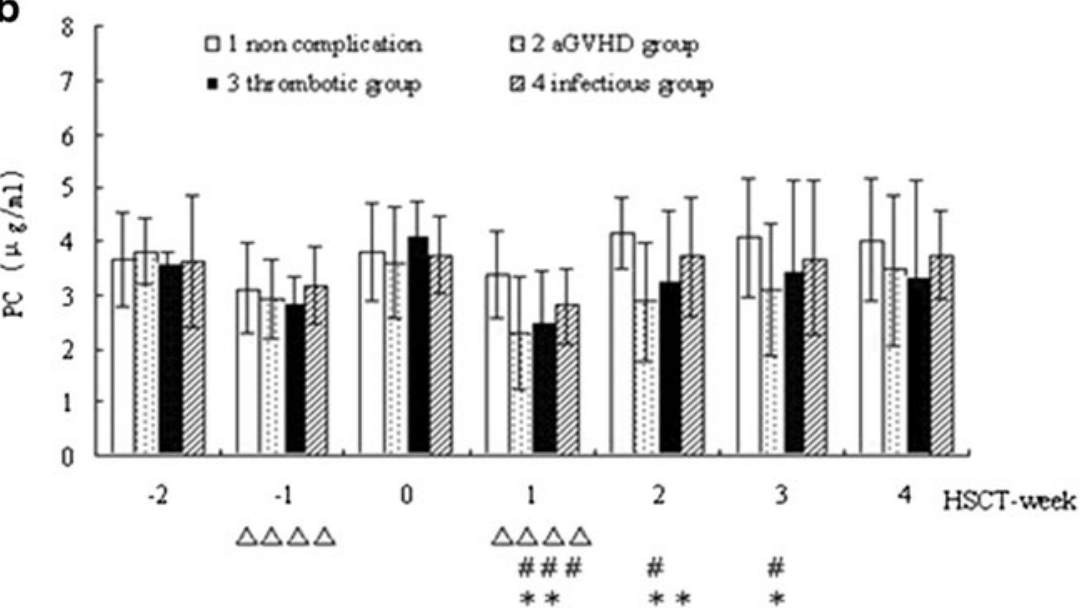

$(P<0.05)$ followed by a small increase immediately after transplantation (week 0). After engraftment, plasma PC of all patients showed the lowest levels in the first week of transplantation and then returned to normal range. Patients with complications had lower plasma PC levels compared with those without complication after transplantation $(P<0.05)$, of which patients with aGVHD showed the lowest PC level (Fig. 1b).

Maximum levels of plasma PAI-1 and t-PA and minimum level of PC in patients receiving HSCT in the early phase of TRCs

During transplantation, patients were grouped based on the first-onset complications following allogeneic HSCT. Of 107 patients, 38 presented with aGVHD, 28 with infection, 7 with thrombotic complication, and 34 without complication. When plasma levels of PAI-1, PC, and t-PA were analyzed using peak values, patients with complications showed higher maximum plasma PAI-1 levels than those without complications during the observation weeks $(P<$ 0.05). Maximum values of PAI-1 in all patients with thrombotic complications were above the normal range of plasma PAI-1 $(5-45 \mathrm{ng} / \mathrm{ml})(7 / 7,100 \%)$, while 16 cases in the aGVHD group $(16 / 38,42.1 \%)$ and 15 cases in the infection group $(14 / 28,50.0 \%)$ showed abnormal maximum levels of PAI-1 antigen. Moreover, the peak PAI-1 antigen levels were even higher in seven patients with thrombotic complications than those in patients with other TRCs (thrombosis, $62.77 \pm 7.46 \mathrm{ng} / \mathrm{ml}$; aGVHD, $44.12 \pm$ $8.16 \mathrm{ng} / \mathrm{ml}, P<0.05$; infection, $47.95 \pm 9.19 \mathrm{ng} / \mathrm{ml}, P<0.05$ ) (Fig. 2a). On the contrary, all the patients with three different complications presented with significantly low minimum level of protein $\mathrm{C}$ in comparison with those without complications $(P<0.05)$. The minimum PC levels were apparently lower in the GVHD patients than those in patients with other TRCs (aGVHD, $1.87 \pm 0.52 \mu \mathrm{g} / \mathrm{ml}$; thrombosis, $2.38 \pm 0.55 \mu \mathrm{g} / \mathrm{ml}$; infection, $2.67 \pm 0.45 \mu \mathrm{g} / \mathrm{ml}$. $P<0.05$ ) (Fig. 2b). In addition, the maximum t-PA antigen levels in seven patients with thrombotic complications were significantly higher than those in patients with other complications in the course of the investigation (thrombosis, $33.33 \pm 6.11 \mathrm{ng} / \mathrm{ml}$; aGVHD, $18.92 \pm 6.33 \mathrm{ng} / \mathrm{ml}$; infection, $13.82 \pm 5.75 \mathrm{ng} / \mathrm{ml} . \quad P<0.05)$. There was no statistical 
Fig. 2 Maximum plasma PAI-1, $\mathrm{t}-\mathrm{PA}$, and minimum $\mathrm{PC}$ in patients with TRCs following HSCT. a Gray background shows the normal range of plasma PAI-1 antigen levels (5-45 ng/ml), marks indicate the maximum PAI-1 antigen levels, and bars show the $95 \%$ CI. b Gray background shows the normal range of plasma PC antigen levels $(3.0-5.2 \mu \mathrm{g} / \mathrm{ml})$. Marks indicate the minimum plasma PC antigen level, and bars show the $95 \%$ CI. c Gray background shows the normal range of plasma t-PA antigen levels (1.0-12 ng/ml). Marks indicate the maximum t-PA antigen levels, and bars show the $95 \%$ CL. $(\triangle P<0.05$ : compared to the thrombotic group, $\boldsymbol{\nabla} P<0.05$ : compared to the GVHD group, $\# P<0.05$ : compared to the infectious group)
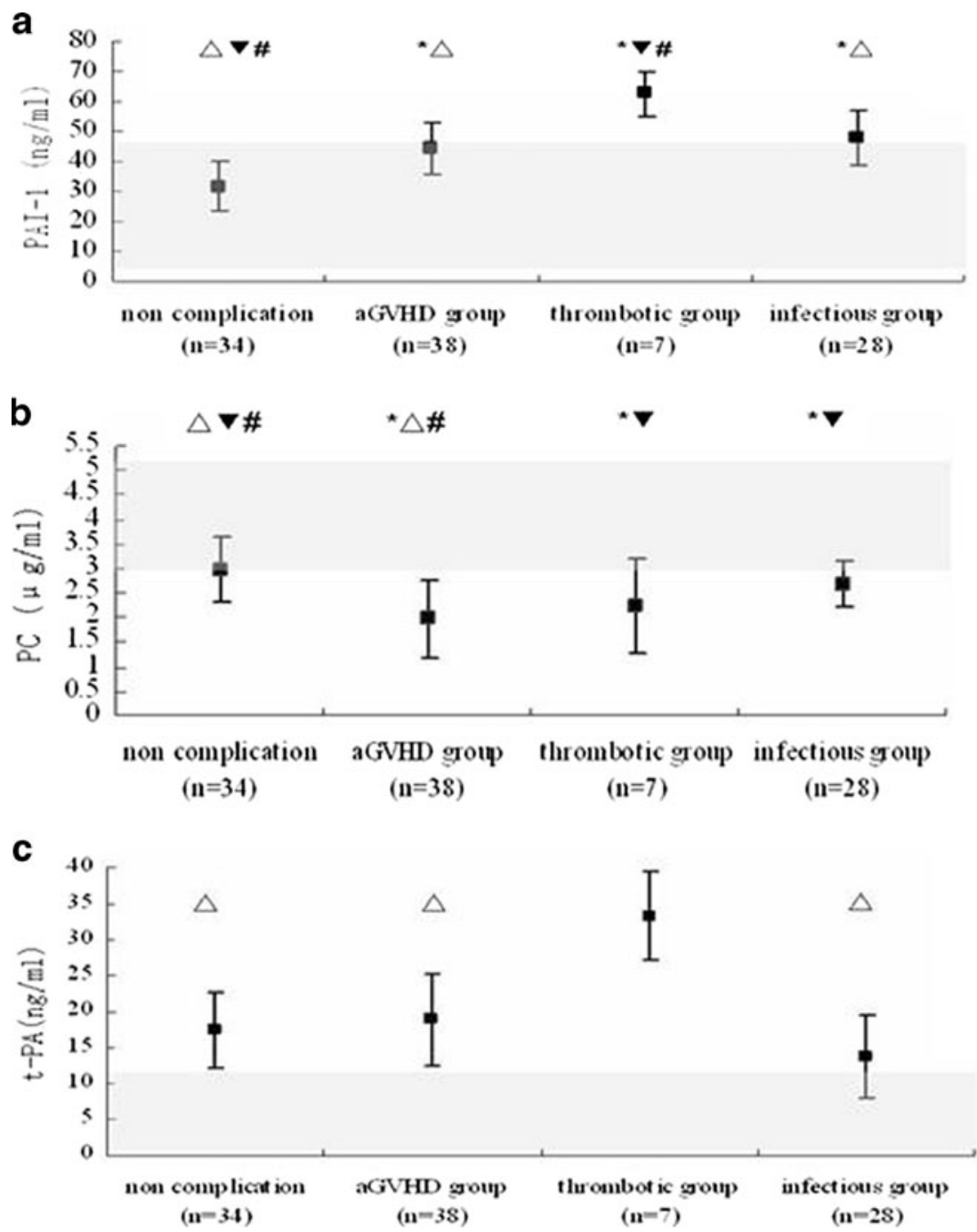

difference in the peak t-PA antigen levels among the patients with aGVHD and infection, and those without complications (Fig. 2c).

\section{Discussion}

Thrombotic events closely related to endothelium injuries and coagulation disturbance are severe and life-threatening complications in allogeneic HSCT recipients. The incidences of the most common two types, VOD (frequency, 1-54\%) [8] and TA-TMA (frequency, 0.5-63.6\%) [22], vary greatly according to different criteria. Our observation showed that eight patients developed thrombotic complications in 107 patients following allogeneic HSCT (7.5\%), including six VOD, one TA-TMA, and one DVT. The incidences of VOD $(5.6 \%)$ and TA-TMA $(0.9 \%)$ were in the lower range of other reports $[8,22]$, probably due to the difference in race or diagnostic criteria for TA-TMA.
The low specificity and late onset of diagnostic clinical signs of thrombotic complications hinder their early recognition and differential diagnosis from other TRCs. Since the early and rational treatments can improve outcomes for patients affected by these complications of HSCT, early diagnosis of thrombotic events is essential [23, 24]. We have determined some predictive laboratory markers for the differential diagnosis of early TRCs and performed a systemic survey of hemostatic parameters in allogeneic HSCT recipients. The most notable findings were the dramatic alterations of plasma PAI-1 and protein $\mathrm{C}$ levels in the patients with TRCs.

PAI-1, a physiologic antagonist of t-PA, mainly synthesized and released by endothelial cells [25], is the major inhibitor of the fibrinolytic enzyme cascade [26]. It has been proposed to be an early marker of VOD diagnosis both in pediatric and adult HSCT patients [11, 12]. PAI-1 inhibitor could prevent hepatic venous injury in murine models, and PAI-1-deficient mice were protected largely 
from VOD [27]. Some investigators also reported an elevated level of PAI-1 or t-PA/PAI in TA-TMA [14, 15]. We found a significant elevation of mean plasma PAI-1 antigen in the patients with complications before and after 2 weeks of stem cell transfusion, with which a most significant increase in plasma PAI-1 occurred in patients with thrombotic complication throughout the observation period. This result was similar to the findings of others [1115], demonstrating an endothelial injury by conditioning, transplantation complications, and the activation of the fibrinolytic system. Most importantly, the evidence that the peak plasma PAI-1 antigen levels in our studies presented in patients with complications suggested that the maximum PAI-1 plasma level could be a useful predictive marker for the early diagnosis of TRCs in HSCT patients. Moreover, the highest plasma PAI-1 level in patients with thrombotic complication could be used to discriminate thrombotic events from other TRCs in allogeneic HSCT patients [11]. In addition, our data also showed a significant higher level of maximum plasma t-PA antigen only in the thrombotic group, implying that peak t-PA antigen could be used in combination with PAI-1 to predict the early diagnosis of thrombotic complications.

Protein C plays an important role in preventing blood clot formation in vivo. Upon activation, PC functions as an anticoagulant by inactivating factors Va and VIIIa [28]. Several reports analyzed PC and another natural anticoagulant ATIII to define their values in the diagnosis of TRCs [29]. However, the results were controversial. Some studies suggested that the decrease of plasma PC or ATIII reflects the development of VOD [29, 30], portal vein thrombosis [31], or early occurrence of TRCs [18], while others could not verify their roles in the differential diagnosis of TRCs $[14,16]$. We observed the diminution of mean and minimum plasma PC levels in the patients with TRC after HSCT, indicating the correlation of plasma PC level with the development of TRCs. Furthermore, the lowest levels of mean and minimum PC were observed in the patients with aGVHD, suggesting the early post-BMT reduction of PC might predict the occurrence of aGVHD.

Although other studies showed many subclinical clotting and fibrinolytic profile changes during the period following conditioning chemotherapy and the period after transplantation [32], we did not find any significant change in the parameters of APTT, AT, PT, and D-Di, probably owing to the modified treatment or the heterogeneity of Chinese patients.

We also grouped patients based on single or multiple complications. We found that the peak PAI-1 antigen levels were higher in patients with only thrombotic complication than those with other single TRCs. The minimum PC levels were apparently lower in the aGVHD patients than those in patients with other single TRCs. The maximum t-PA antigen levels in patients with only thrombotic complication were significantly higher than those in patients with other single complications. These results were consistent with those obtained when patients were grouped by the first onset of complications.

In summary, alteration of hemostatic parameters during the early phase of HSCT reflects endothelial injury or a hypercoagulable state in patients undergoing allogeneic HSCT, which contributes to the development of TRCs. The increase in plasma PAI-1 levels, particularly in combination with increased t-PA level, can be used as a marker for the early discrimination of thrombotic complication from other TRCs, while the reduction of PC might be considered as a good parameter to the early diagnosis of aGVHD. These findings would be useful in improving the early discrimination and correct treatment of TRCs.

Acknowledgments This work was supported by grants from the Jiangsu Province of China (BK2007508, SH201024, and 09KJB320014) and funded by the Priority Academic Program Development of the Jiangsu Higher Education Institutions (PAPD).

Conflict of interest The authors have no conflict of interest to declare.

Open Access This article is distributed under the terms of the Creative Commons Attribution Noncommercial License which permits any noncommercial use, distribution, and reproduction in any medium, provided the original author(s) and source are credited.

\section{References}

1. Santos GW, Tutschka PJ, Brookmeyer R et al (1983) Marrow transplantation for acute nonlymphocytic leukemia after treatment with busulfan and cyclophosphamide. N Engl J Med 309:1347-1353

2. Thomas ED, Clift RA, Fefer A et al (1986) Marrow transplantation for the treatment of chronic myelogenous leukemia. Ann Intern Med 104:155-163

3. Appelbaum FR, Sullivan KM, Buckner CD et al (1987) Treatment of malignant lymphoma in 100 patients with chemotherapy, total body irradiation, and marrow transplantation. J Clin Oncol $5: 1340-1347$

4. Petersdorf EW, Gooley TA, Anasetti C et al (1998) Optimizing outcome after unrelatd marrow transplantation by comprehensive matching of HLA class I and II alleles in the donor and recipient. Blood 92:3515-3520

5. Woolfrey AE, Anasetti C, Storer B et al (2002) Factors associated with outcome after unrelated marrow transplantation for treatment of acute lymphoblastic leukaemia in children. Blood 99:2002-2008

6. Cutler C, Giri S, Jeyapalan S et al (2001) Acute and chronic graft versus host disease after allogeneic peripheral blood stem cell and bone marrow transplantation: a meta-analysis. J Clin Oncol 19:3685-3691

7. Qu L, Kiss JE (2005) Thrombotic microangiopathy in transplantation and malignancy. Semin Thromb Hemost 31:691-699

8. Bearman SI (1995) The syndrome of hepatic veno-occlusive disease after marrow transplantation. Blood 85:3005-3020

9. Kearon C, Ginsberg JS, Hirsh J (1998) Venus thromboembolic disease. In: Yusuf S, Cairns JA, Camm AJ, Fallen EL, Gersh BJ 
(eds) Evidence based cardiology. BMJ Publishing Group, London, pp 1009-1024

10. Nadir Y, Brenner B (2007) Hemorrhagic and thrombotic complications in bone marrow transplant recipients. Thromb Res 120: S92-S98

11. Pihusch M, Wegner H, Goehring $P$ et al (2005) Diagnosis of hepatic veno-occlusive disease by plasminogen activator inhibitor1 plasma antigen levels: a prospective analysis in 350 allogeneic hematopoietic stem cell recipients. Transplantation 80:1376-1382

12. Sartori MT, Spiezia L, Cesaro S et al (2005) Role of fibrinolytic and clotting parameters in the diagnosis of liver veno-occlusive disease after hematopoietic stem cell transplantation in a pediatric population. Thromb Haemost 93:682-689

13. Salat C, Holler E, Kolb HJ et al (1997) Plasminogen activator inhibitor-1 confirms the diagnosis of hepatic veno-occlusive disease in patients with hyperbilirubinemia after bone marrow transplantation. Blood 89:2184-2188

14. Kanamori H, Maruta A, Sasaki S et al (1998) Diagnostic value of hemostatic parameters in bone marrow transplant-associated thrombotic microangiopathy. Bone Marrow Transplant 21:705-709

15. Anthony MT, Zeigler ZR, Lister J et al (1998) Plasminogen activator inhibitor (PAI-1) antigen levels in primary TTP and secondary TTP post-bone marrow transplantation. Am J Hematol 59:9-14

16. Pihusch M, Wegner H, Goehring $P$ et al (2005) Protein $C$ and procollagen III peptide levels in patients with hepatic dysfunction after allogeneic hematopoietic stem cell transplantation. Bone Marrow Transplant 36:631-637

17. Tamaki S, Wada H, Ohfuzi K et al (2002) Hemostatic abnormalities following bone marrow transplantation. Clin Appl Thromb Hemost 8:125-132

18. Klingebiel T, Schlegel PG (1998) GVHD: overview on pathophysiology, incidence, clinical and biological features. Bone Marrow Transplant 21:S45-S49

19. McDonald GB, Hinds MS, Fisher LD et al (1993) Veno-occlusive disease of the liver and multiorgan failure after bone marrow transplantation: a cohort study of 355 patients. Ann Intern Med 118:255-267

20. Ho VT, Cutler C, Carter S et al (2005) Blood and marrow transplant clinical trials network toxicity committee consensus summary: thrombotic microangiopathy after hematopoietic stem cell transplantation. Biol Blood Marrow Transplant 11:571-575
21. Nichols WG (2003) Management of infectious complications in the hematopoietic stem cell transplant recipient. J Intensive Care Med 18:295-312

22. George JN, Li X, McMinn JR et al (2004) Thrombotic thrombocytopenic purpura-hemolytic uremic syndrome following allogeneic HPC transplantation: a diagnostic dilemma. Transfusion 44:294-304

23. Richardson PG, Murakami C, Jin Z et al (2002) Multi-institutional use of defibrotide in 88 patients after stem cell transplantation with severe venoocclusive disease and multisystem organ failure: response without significant toxicity in a high-risk population and factors predictive of outcome. Blood 100:4337-4343

24. Batts ED, Lazarus HM (2007) Diagnosis and treatment of transplantation-associated thrombotic microangiopathy: real progress or are we still waiting? Bone Marrow Transplant 40:709-719

25. Kruithof EK (1988) Plasminogen activator inhibitors-a review. Enzyme 40:113-121

26. Wiman B, Chmielwska J, Rånby M (1984) Inactivation of tissue plasminogen activator in plasma. Demonstration of a complex with a new rapid inhibitor. J Biol Chem 259:3644-3647

27. Smith LH, Dixon JD, Stringham JR et al (2006) Pivotal role of PAI-1 in a murine model of hepatic vein thrombosis. Blood 107:132-134

28. Esmon CT (1987) The regulation of natural anticoagulant pathways. Science 235:1348-1352

29. Faioni EM, Krachmalnicoff A, Bearman SI et al (1993) Naturally occurring anticoagulants and bone marrow transplantation: plasma protein $\mathrm{C}$ predicts the development of venocclusive disease of the liver. Blood 81:3458-3462

30. Lee JH, Lee KH, Kim S et al (1998) Relevance of proteins C and $\mathrm{S}$, antithrombin III, von Willebrand factor, and factor VIII for the development of hepatic veno-occlusive disease in patients undergoing allogeneic bone marrow transplantation: a prospective study. Bone Marrow Transplant 22:883-888

31. Kikuchi K, Rudolph R, Murakami C et al (2002) Portal vein thrombosis after hematopoietic cell transplantation: frequency, treatment and outcome. Bone Marrow Transplant 29:329-333

32. Notoya A, Sawada K, Ieko M et al (1998) APBSCT: subclinical alterations in coagulation and fibrinolysis in patients undergoing autologous peripheral blood stem cell transplantation. Leuk Lymphoma 28:405-413 